\title{
BMJ Open Utilisation, expenditure and cost-effectiveness of cystic fibrosis drugs in Ireland: a retrospective analysis of a national pharmacy claims database
}

Amelia Smith (D) , ${ }^{1,2}$ Michael Barry ${ }^{1,2,3}$

To cite: Smith A, Barry M. Utilisation, expenditure and cost-effectiveness of cystic fibrosis drugs in Ireland: a retrospective analysis of a national pharmacy claims database. BMJ Open 2020;10:e040806. doi:10.1136/ bmjopen-2020-040806

- Prepublication history for this paper is available online. To view these files, please visit the journal online (http://dx.doi. org/10.1136/bmjopen-2020040806).

Received 22 May 2020 Revised 19 0ctober 2020 Accepted 20 0ctober 2020

D) Check for updates

(C) Author(s) (or their employer(s)) 2020. Re-use permitted under CC BY-NC. No commercial re-use. See rights and permissions. Published by BMJ.

${ }^{1}$ Department of Pharmacology and Therapeutics, Trinity Centre for Health Sciences, St James's Hospital, University of Dublin Trinity College, Dublin, Ireland ${ }^{2}$ Medicines Management Programme, Health Service Executive, Dublin, Ireland

${ }^{3}$ National Centre for

Pharmacoeconomics, St. James's Hospital, Dublin, Ireland

Correspondence to

Dr Amelia Smith;

smitha25@tcd.ie

\section{ABSTRACT}

Objectives To determine the use and expenditure associated with cystic fibrosis (CF) modulator therapies in Ireland since their reimbursement in 2013.

Design A retrospective analysis of a national drug claims database.

Setting The data included in this study are nationally representative (Ireland).

Participants Data on all persons receiving CF modulator therapies were included.

Methods We obtained national claims data for $\mathrm{CF}$ therapies from the Health Service Executive's Primary Care Reimbursement Service. We determined the use and expenditure associated with CF therapies from January 2012 to March 2020.

Results The increased prescribing of CF modulator therapies was associated with an approximate fivefold increase in expenditure from $€ 23$ million in 2013 to $€ 113$ million in 2019. Many patients who initiated lumacaftor/ ivacaftor in 2017 went on to receive symptomatic therapies, and subsequently initiated tezacaftor/ivacaftor in 2019.

Conclusion Despite none of these modulator therapies demonstrating value for money when subjected to health technology assessment, the associated Irish expenditure reached $€ 113$ million in 2019 alone.

\section{INTRODUCTION}

Cystic fibrosis $(\mathrm{CF})$ is an inherited autosomal recessive condition caused by mutations in the gene encoding the $\mathrm{CF}$ transmembrane conductance regulator (CFTR) protein leading to reduced CFTR function. ${ }^{1}$ CFTR codes for an epithelial anion channel that transports chloride and bicarbonate across epithelial surfaces in areas such as the respiratory tract, pancreas, gastrointestinal system and sweat glands, resulting in clinical manifestations characterised by pancreatic insufficiency and chronic lung disease with progressive loss of lung function, repeated pulmonary exacerbations and increased mortality. ${ }^{2}$ Molecularly targeted therapies called CFTR modulators target specific

\section{Strengths and limitations of this study}

- This study is one of the first to describe nationwide use and expenditure associated with the novel cystic fibrosis (CF) modulator therapies.

- The use of a robust claims database provides an opportunity to elicit longitudinal State spending on CF modulator therapies.

- Unfortunately, the database does not include patient characteristics or patient outcomes.

defects caused by mutations in CFTR thereby treating the underlying cause of $\mathrm{CF}$.

The first of the CFTR modulators was ivacaftor (Kalydeco), a 'potentiator' which increases channel gating of CFTR enhancing chloride ion transport. ${ }^{3}$ These new therapies that target the underlying cause of $\mathrm{CF}$ were much awaited by $\mathrm{CF}$ patients, given the lack of targeted therapies available. These drugs are particularly relevant to the Irish and UK healthcare systems as Ireland and the UK have the highest incidence rates of $\mathrm{CF}$ in the world. ${ }^{4}$ A study by Farrell determined the incidence of $\mathrm{CF}$ in the Irish population at 1:1353; the highest among Western European and North American countries. ${ }^{4}$ An analysis of the Irish CF Registry has shown that the direct costs associated with CF have increased over the last number of years, with inpatient bed-day costs increasing from $€ 14026$ in 2008 to $€ 17332$ in 2012 , and medication costs increasing from $€ 5863$ in 2008 to $€ 12467$ in $2012 .^{5}$ Vertex Pharmaceuticals, through a 'venture philanthropy' partnership with the American Cystic Fibrosis Foundation, hold the licence in the US and Europe, availing of orphan drug legislation. ${ }^{6}$ In 2013, the National Centre for Pharmacoeconomics (NCPE) conducted a health technology assessment (HTA) of ivacaftor and determined that reimbursement would pose a significant budget impact to the 
State, at a cost of over $€ 28$ million each year or $40 \%$ of the funding available for all new drug treatments in that year. However, in March 2013, an agreement was reached between the Health Service Executive (HSE) and Vertex Pharmaceuticals and the drug was subsequently approved from reimbursement.

Following ivacaftor came 'corrector' therapies such as lumacaftor, tezacaftor and elexacaftor, which increase the amount of functional mutated CFTR. ${ }^{3}$ Following some degree of media controversy as a result of a negative HTA from the NCPE, lumacaftor/ivacaftor (Orkambi) was reimbursed in 2017. ${ }^{7}$ Tezacaftor/ivacaftor (Symkevi) was reimbursed in 2019; however, this drug was not subjected to HTA and was incorporated into a so-called 'portfolio deal' with the manufacturer. ${ }^{8}$ Finally, the triplecombination regimen of elexacaftor, tezacaftor and ivacaftor (Trikafta), is currently pending European Medicines Agency approval.

These CFTR modulators are reimbursed through the HSE Primary Care Reimbursement Service (PCRS). Specifically, the high-tech (HT) drug arrangement, whereby the State facilitates access to certain high-cost medicines through community pharmacies. The aims of this paper are:

- to outline the use and expenditure associated with the CFTR modulators in the Irish healthcare setting since their introduction in 2013.

- determine the symptomatic treatment burden in patients receiving CFTR modulators.

- review the NCPE HTA reports, which outline the costeffectiveness of the CFTR modulators.

\section{METHODS}

This study was carried out using data from the PCRS HT drugs arrangement claims database. HT medicines are purchased by the HSE and supplied through community pharmacies for which pharmacists are paid a patient care fee, with the cost of the medicines and patient care fees being paid by the PCRS. Once a product is approved for reimbursement under the HT drugs arrangement, it is given a unique five-digit code. This code is collected in the HT claims database and is used to determine the number of products dispensed and number of patients in receipt of these products. Unfortunately, there is no other patient information (such as age, gender and clinical characteristics) or outcome data collected in the database. As these data are collected for the purpose of claims reimbursement, missing data are negligible. In Ireland, persons with $\mathrm{CF}$ who are 'ordinarily resident' can receive their medicines free of charge, without means testing, under the HT drugs arrangement. Therefore, it is reasonable to assume that the claims data included in this study are representative of the full CF population. As the first CFTR modulator was approved for reimbursement in 2013, we extracted data from January 2012 to March 2020 for the following drugs; Kalydeco (ivacaftor), Orkambi (lumacaftor/ivacaftor), Symkevi (tezacaftor/ivacaftor), Pulmozyme (dornase alfa), nebulised tobramycin, aztreonam and colistimethate. Using the HT claims database, we investigated the following:

- the use of ivacaftor, lumacaftor/ivacaftor, tezacaftor/ ivacaftor, dornase alfa, nebulised tobramycin, aztreonam and colistimethate in terms of number of prescriptions, and number of patients.

- the State expenditure associated ivacaftor, lumacaftor/ ivacaftor, tezacaftor/ivacaftor, dornase alfa, nebulised tobramycin, aztreonam and colistimethate in terms of ingredient cost (cost to the HSE, excluding Valueadded Tax (VAT) and patient care fees).

- the use of dornase alfa, nebulised tobramycin, aztreonam and colistimethate in patients who initiated ivacaftor in 2013.

- the use of tezacaftor/ivacaftor, dornase alfa, nebulised tobramycin, aztreonam and colistimethate in patients who initiated lumacaftor/ivacaftor in 2017.

To determine the use of symptomatic therapies in patients initiating either ivacaftor in 2013, or lumacaftor/ ivacaftor in 2017, we used an individual patient identifier to extract all claims data for those patients (until March 2020). We used Poisson linear regression to test for trend over time. Significance at $\mathrm{p}<0.05$ is assumed.

We also conducted a review of cost-effectiveness reports in relation to ivacaftor and lumacaftor/ivacaftor, which were obtained from published HTA reports by the NCPE. ${ }^{9-12}$ HTA reports present data such as clinical outcomes, incremental cost-effectiveness ratio (ICER) in terms of cost per quality adjusted life year (cost/QALY) and the estimated 5-year budget impact. Drug use and expenditure data were extracted from the HT claims database and imported into SAS V.9.4 software.

\section{Patient and public involvement}

Patients and/or the public were not involved in the design, or conduct, or reporting, or dissemination plans of this research.

\section{RESULTS}

The prescribing of CF modulator therapies increased eightfold over the time period 2012-2019, from a prescribing frequency of 1192-9550 prescriptions per annum associated with the increasing therapeutic indications for ivacaftor and the introduction of combination therapies such as lumacaftor/ivacaftor and tezacaftor/ ivacaftor (figure 1). The number of patients receiving symptomatic therapies including dornase alfa, nebulised tobramycin, aztreonam and colistimethate remained largely unchanged. There was an average of 354 patients in receipt of dornase alfa in 2013, 423 patients in 2017 and 430 patients in 2019. There was an average of 177 patients receiving tobramycin and/or aztreonam in 2013, 210 patients in 2017 and 191 patients in 2019.

The increased prescribing of $\mathrm{CF}$ modulator therapy was associated with an approximate fivefold increase in expenditure from $€ 23.1$ million in 2013 to $€ 113.7$ million in 2019 (figure 2). In 2019 alone, $€ 44105608$ was 


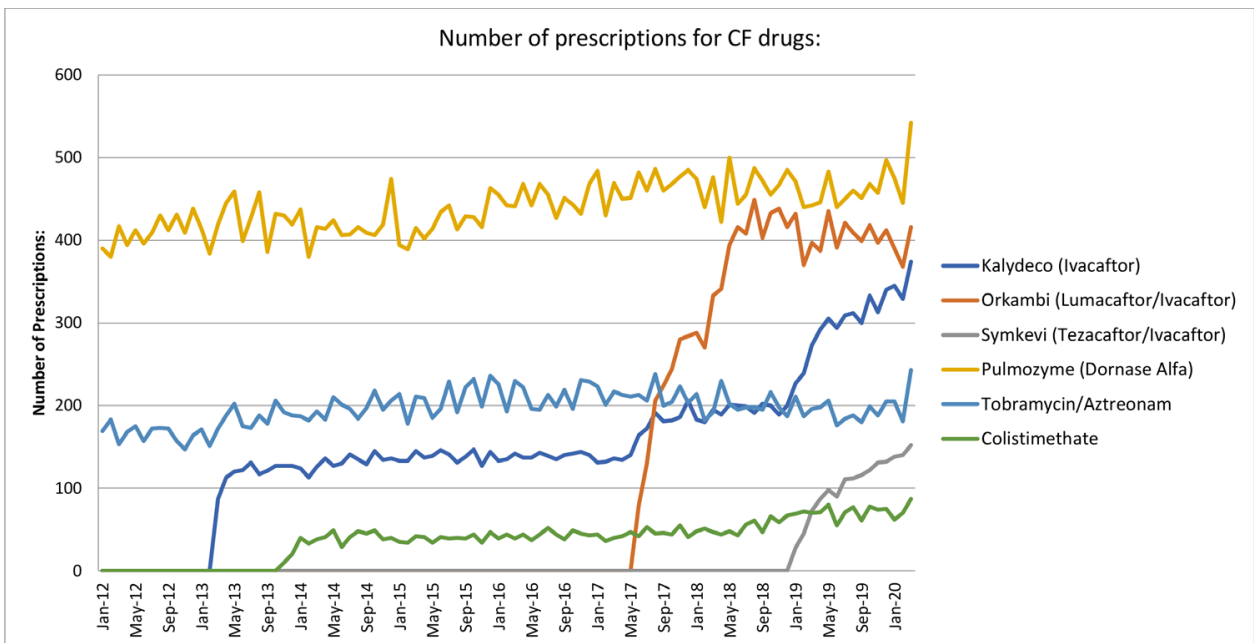

Figure 1 The number of prescription claims for each of the CF drugs, from January 2012 to March 2020. CF, cystic fibrosis.

spent on ivacaftor, $€ 61072176$ on lumacaftor/ivacaftor and $€ 8536355$ tezacaftor/ivacaftor.

After initiating ivacaftor in $2013(\mathrm{n}=149)$, there was a statistically significant decrease in the number of these patients receiving the symptomatic therapies dornase alfa $(\mathrm{p}<0.001)$ and tobramycin $(\mathrm{p}<0.001)$, as shown in figure 3 . The use of colistimethate was unchanged $(p=0.98)$. There was also a non-significant increase in the number of ivacaftor patients receiving aztreonam $(\mathrm{p}=0.08)$. However, this decline is not observed in patients who initiated lumacaftor/ivacaftor in 2017 (figure 4); the use of tobramycin and aztreonam remained unchanged $(\mathrm{p}=0.45$ and $\mathrm{p}=0.37$, respectively) There was a non-significant decrease in the number of lumacaftor/ivacaftor patients receiving dornase alfa $(p=0.08)$. There was also a statistically significant increase in the number of patients receiving colistimethate $(p=0.01)$. Interestingly, the number of patients $(\mathrm{n}=82)$ who initiated lumacaftor/ivacaftor in 2017 , went on to receive tezacaftor/ivacaftor on its introduction in 2019.

The cost-effectiveness of ivacaftor was assessed in 2013, 2016 and 2017 for three-patient cohorts; patients aged 6 years and older who have the G551D mutation, patients aged 2 years and older and weighing less than $25 \mathrm{~kg}$ who have one of nine gating (class III) mutations in the CFTR gene, and patients aged 18 years and older who have the $\mathrm{R} 117 \mathrm{H}$ mutation in the CFTR gene, respectively. At the submitted asking price of $€ 234803$ per patient per annum, ivacaftor was not cost-effective for any of the three-patient cohorts with the ICER exceeding $€ 400000 /$ QALY in each case. The estimated 5-year gross budget impact for the three ivacaftor indications exceeded $€ 120$ million. The costeffectiveness of lumacaftor/ivacaftor for patients aged 12 years and older who are homozygous for the F508del mutation in the CFTR gene was assessed in June 2016. At an asking price of $€ 158306$ per patient per annum, it was not cost-effective with an ICER of €649 624/QALY and an estimated 5-year budget impact of $€ 391$ million. Tezacaftor/ivacaftor was not subjected to HTA prior to its reimbursement in 2019 due to the introduction of a 'portfolio deal'. The 'portfolio deal' was agreed in June 2017 , and it states 'this innovative long-term agreement enables rapid access for people with these mutations if the labels of the existing medicines are expanded to cover additional age groups and if new Vertex medicines are approved for these populations'.

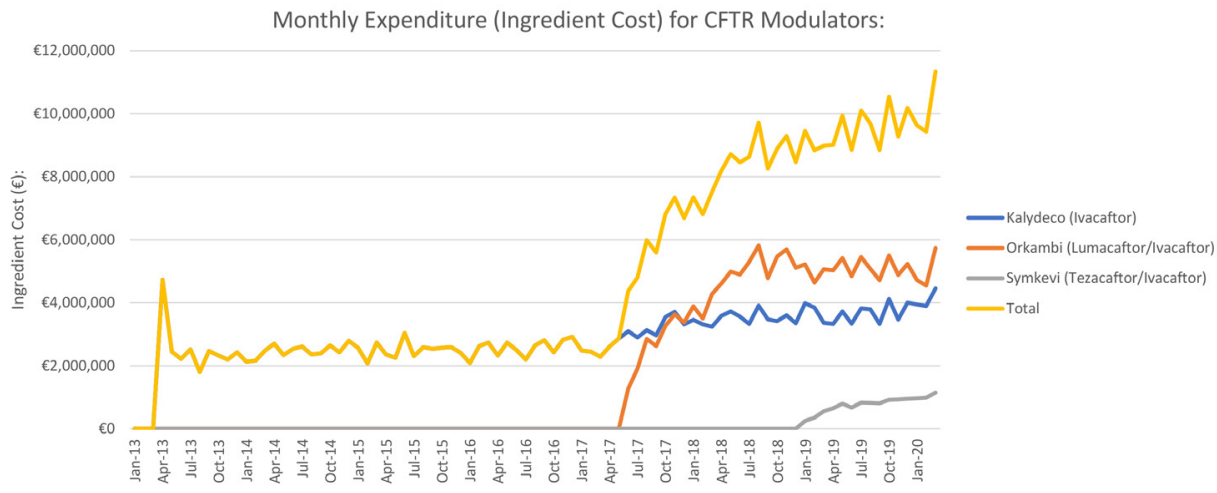

Figure 2 The monthly expenditure associated with each cystic fibrosis drug, based on ingredient cost, from January 2012 to March 2020. CFTR, cystic fibrosis transmembrane conductance regulator. 


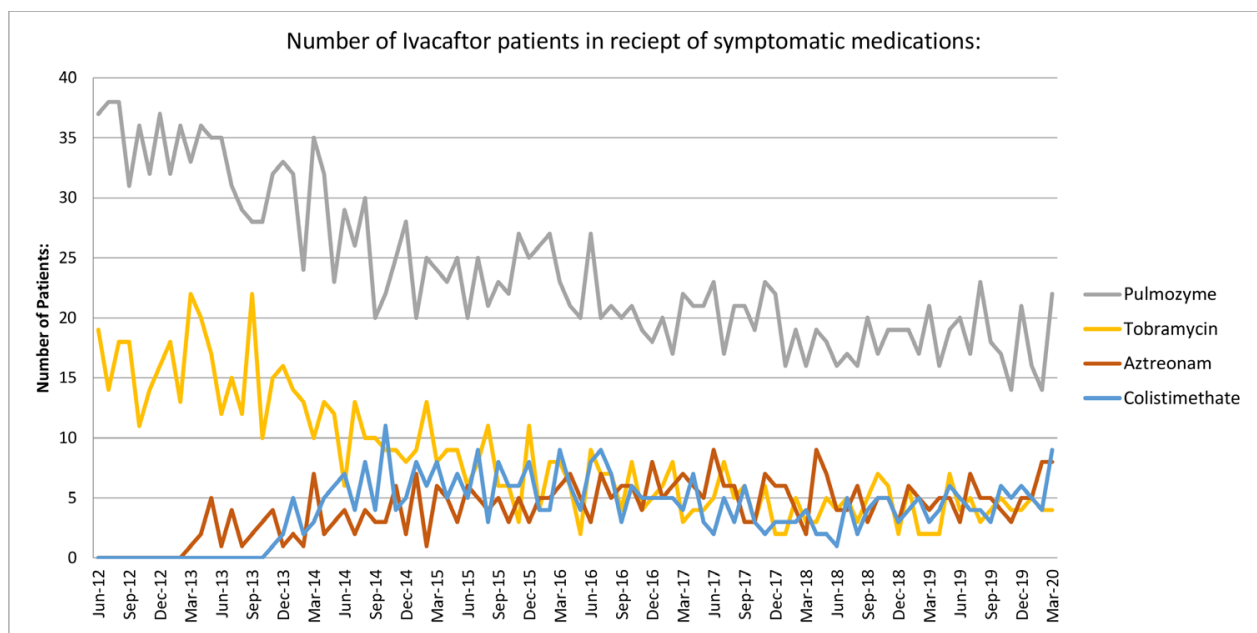

Figure 3 The number of patients (from cohort of patients initiating ivacaftor in 2013, n=149) in receipt of symptomatic medications.

\section{DISCUSSION}

As of March 2020, there are 339 patients being treated with ivacaftor, which was the first $\mathrm{CF}$ modulator to be reimbursed in Ireland in 2013. This followed the demonstration that ivacaftor improved lung function in patients 12 years or older with at least one G551D-CFTR mutation. ${ }^{13}$ Low patient numbers, the short duration of the clinical trial at 48 weeks and the use of a surrogate marker (per cent predicted forced expiratory volume in 1 second $\left(\mathrm{ppFEV}_{1}\right)$ ) for the primary endpoint were recognised limitations and long-term data are required to demonstrate an impact on the deterioration in pulmonary function. Despite the beneficial effects on ppFEV1, many patients who receive ivacaftor continue to need other treatments to control CF such as pancreatic enzyme replacement, inhaled mucolytic drugs and antibiotic therapies. ${ }^{14}$ This is reflected in our prescribing data.

The asking price for ivacaftor was $€ 234803$ per patient per year and it was subjected to three NCPE HTAs from 2013 to 2017 to determine its value for money. The first HTA report on the use of ivacaftor for the treatment of CF patients aged 6 years or older who had the G551D mutation was published on the 1 January 2013.
It concluded that ivacaftor was not cost-effective with an ICER of $€ 449$ 035/QALY, which exceeds the Irish cost-effectiveness threshold of $€ 45000 /$ QALY. The estimated 5-year budget impact was just over $€ 28$ million with approximately 120 eligible patients. Ivacaftor was subjected to two further HTAs; for patients with CF aged 2 years or older who had one of nine gating mutations and, for CF patients 18 years or older with the R117H mutation. The drug was not considered cost-effective for either therapeutic indication with ICERs of $€ 465$ 546/ QALY and $€ 444466 /$ QALY, respectively. The estimated budget impact for these indications is $€ 90$ million over the next 5 years. A US study by Dilokthornsakul et al estimated the lifetime costs of ivacaftor to be approximately $\$ 3.4$ million higher than usual care alone. However, as $\mathrm{CF}$ is a rare disease that only affects about 30000 patients (and even fewer with G551D mutation) in the USA, the cost may be tolerated by some US payers. ${ }^{15}$

Our study does show a reduction in the use of symptomatic treatments in patients who commenced ivacaftor in 2013. An Irish longitudinal study has investigated trends in health outcomes following the initiation of ivacaftor; in the 36 months following ivacaftor therapy,

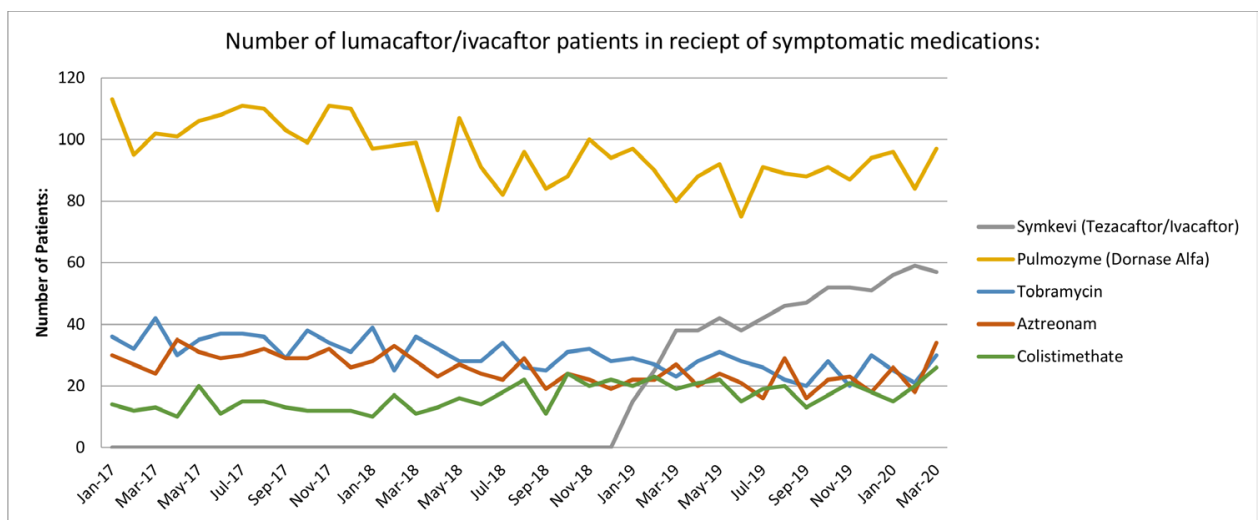

Figure 4 The number of patients (from cohort of patients initiating lumacaftor/ivacaftor in 2017, n=345) in receipt of symptomatic medications. 
the ppFEV1 improved by $2.26 \%$ per year $(95 \% \mathrm{CI}, 0.2$ to 4.3) for patients aged younger than 12 years, remained unchanged for $12-18$ years old (95\% CI, -1.9 to 2.9 ), and declined in adults by $1.74 \%$ per year (95\% CI, 23.1 to 20.4). Intravenous antibiotic use decreased by $46 \%$ and oral antibiotic use decreased by $49 \%$ in the year after ivacaftor initiation. This longitudinal study noted that improvement in clinical outcomes after ivacaftor initiation occurs in the context of concomitant long-term $\mathrm{CF}$ symptomatic therapy use, and there was frequent macrolide and mucolytic use at baseline. However, there was no significant reduction in hospitalisations following ivacaftor therapy. ${ }^{16}$ Five-year follow-up data from CF patient registries suggest that ivacaftor-treated patients had better preserved lung function, improved nutritional status and a lower frequency of pulmonary exacerbations and hospitalisations. ${ }^{14} 17$ Up to $90 \%$ of people with CF require pancreatic enzyme replacement therapy to prevent malnutrition, and as data on these products are not collected in the HT claims database, we could not investigate their use in the current study. However, the PCRS publishes top-level information on products dispensed via their 'Reporting and Open Data' portal, and suggests that expenditure on these products was $€ 112000$ in May 2020 . $^{18}$

Lumacaftor/ivacaftor is licensed for the treatment of $\mathrm{CF}$ in patients aged 12 years and older who are homozygous for the F508del mutation in the CFTR gene, and as of March 2020, there are 384 patients being treated. The pivotal clinical trials (TRAFFIC and TRANSPORT) reported the mean absolute improvement in the primary endpoint (ppFEV1) ranged from $2.6 \%$ to $4 \%$ compared with placebo over 24 weeks. ${ }^{19}$ A possible reason for the relatively poor response was the combination of a cytochrome P450 3A enzyme inducer (lumacaftor) with ivacaftor, a P450 3A substrate. ${ }^{20}$ In our study, 51 of the 345 patients initiating lumacaftor/ivacaftor in 2017, went on to receive tezacaftor/ivacaftor on its reimbursement in 2019, suggesting that there was inadequate response to lumacaftor/ivacaftor. The drug failed to demonstrate cost-effectiveness as confirmed in the NCPE HTA report published in June 2016. At an asking price of $€ 158306$ per patient per year, the NCPE preferred ICER was $€ 649$ 624/QALY. As the F508del is the most prevalent mutation of the CFTR gene, it was estimated that the 5-year gross budget impact would exceed $€ 391$ million for the 500+ eligible patients. Lumacaftor/ivacaftor was reimbursed in 2017 and an associated expenditure of $€ 132$ million has been recorded to date.

Two clinical trials on the use of tezacaftor/ivacaftor were published in November 2017; treatment of patients with CF homozygous for Phe508del, and for patients heterozygous for the Phe508del and a CFTR mutation associated with residual CFTR function. ${ }^{21}{ }^{22}$ There are 138 patients receiving this treatment as of March 2020. Tezacaftor/ ivacaftor was not subjected to HTA as it was incorporated into a so-called 'portfolio deal' with the manufacturer. ${ }^{8}$ Therefore, there is uncertainty as to the value for money associated with this product, which resulted in an expenditure of $€ 8.5$ million in its first year of reimbursement in 2019. The portfolio deal, in effect, introduced a budget cap for CF modulator therapies with perceived advantages, including immediate patient access, the certainty in relation to budget impact for the payer and the avoidance of previous controversies around CF modulator therapies. However, the HTA process plays an important role in containing pharmaceutical expenditure in Ireland and bypassing this process could have serious long-term financial implications for the healthcare system.

There are several limitations to this study; the data available to us did not include patient characteristics, such as age and gender. The database does not include any efficacy, outcomes or indication data and thus our study cannot consider these. However, to our knowledge, this is the first study to describe the nationwide uptake and expenditure associated with these high-cost and muchawaited CF modulator therapies.

In a recent New England Journal of Medicine editorial, Grasemann states that the clinical efficacy of the current combination $\mathrm{CF}$ modulator therapies for patients with the most common CFTR genotype is suboptimal and differs little from established symptomatic therapies such as nebulised inhaled hypertonic saline or dornase alfa. ${ }^{23}$ Therefore, the focus will now switch to the new CF modulator triple therapy, including the two correctors elexacaftor/tezacaftor plus the potentiator ivacaftor (Trikafta), which has demonstrated efficacy in the treatment of patients with one or two copies of the Phe508del CFTR mutation, raising the prospect of providing $\mathrm{CF}$ modulator therapy for up to $90 \%$ of CF patients. ${ }^{24} 25$

\section{CONCLUSION}

There has been a significant increase in the prescribing of CF modulator therapy in Ireland since 2013. As highlighted in the NCPE HTA reports, none of these therapies demonstrated value for money when subjected to HTA, and expenditure increased to over $€ 113$ million in 2019. As the opportunity cost is running into millions, it is vital that research into longer-term clinical outcomes are realised.

Contributors MB contributed to conception and design, acquisition of data, interpretation of data, drafting the article and final approval of the version to be published. AS contributed to design, analysis and interpretation of data, drafting the article and final approval of the version to be published. MB is the guarantor.

Funding The authors have not declared a specific grant for this research from any funding agency in the public, commercial or not-for-profit sectors.

Competing interests None declared.

Patient consent for publication Not required.

Provenance and peer review Not commissioned; externally peer-reviewed.

Data availability statement Data may be obtained from a third party and are not publicly available. No additional data available.

Open access This is an open access article distributed in accordance with the Creative Commons Attribution Non Commercial (CC BY-NC 4.0) license, which permits others to distribute, remix, adapt, build upon this work non-commercially, and license their derivative works on different terms, provided the original work is 
properly cited, appropriate credit is given, any changes made indicated, and the use is non-commercial. See: http://creativecommons.org/licenses/by-nc/4.0/.

ORCID iD

Amelia Smith http://orcid.org/0000-0003-3070-2268

\section{REFERENCES}

1 Elborn JS. Cystic fibrosis. Lancet 2016;388:2519-31.

2 O'Sullivan BP, Freedman SD. Cystic fibrosis. The Lancet 2009;373:1891-904

3 Collins FS. Realizing the dream of molecularly targeted therapies for cystic fibrosis. N Engl J Med 2019;381:1863-5.

4 Farrell PM. The prevalence of cystic fibrosis in the European Union. $J$ Cyst Fibros 2008;7:450-3.

5 Jackson $A D$, Jackson $A L$, Fletcher $G$, et al. Estimating direct cost of cystic fibrosis care using Irish registry healthcare resource utilisation data, 2008-2012. Pharmacoeconomics 2017;35:1087-101.

6 Ramsey BW, Nepom GT, Lonial S. Academic, Foundation, and industry collaboration in finding new therapies. $N$ Engl J Med 2017;376:1762-9.

7 Praise for campaigners as Orkambi deal agreed with HSE. Available: https://www.irishtimes.com/news/ireland/irish-news/praisefor-campaigners-as-orkambi-deal-agreed-with-hse-1.3045678 [Accessed 10 Aug 2020].

8 Cystic Fibrosis. Landmark decision on Trikafta. Available: https:// www.cfireland.ie/about-cf/latest-news/landmark-decision-on-trikfata [Accessed 10 Aug 2020].

9 NCPE. NCPE ivacaftor (Kalydeco®) assessment, 2013. Available: http://www.ncpe.ie/drugs/ivacaftor-kaldeco/ [Accessed 7 Aug 2020].

10 NCPE. NCPE ivacaftor (Kalydeco®) assessment | ivacaftor (Kalydeco $($ ) for patients with CF 2 years +, National centre for pharmacoeconomics, 2016. Available: http://www.ncpe.ie/drugs/ ivacaftor-kalydeco-for-patients-with-cf-2-years/ [Accessed 7 Aug 2020].

11 NCPE. NCPE ivacaftor (Kalydeco®) assessment | ivacaftor (Kalydeco®) for the treatment of CF patients with the $\mathrm{R} 117 \mathrm{H}$ mutation, National centre for pharmacoeconomics, 2017. Available: http://www.ncpe.ie/drugs/ivacaftor-kalydeco-for-the-treatment-of-cfpatients-with-the-r117h-mutation/ [Accessed 7 Aug 2020].
12 National Centre for Pharmacoeconomics. NCPE Lumacaftor/ ivacaftor (Orkambi®) assessment. Available: http://www.ncpe.ie/ drugs/lumacaftorivacaftor-orkambi/ [Accessed 7 Aug 2020].

13 Ramsey BW, Davies J, McElvaney NG, et al. A CFTR potentiator in patients with cystic fibrosis and the G551D mutation. $N$ Engl J Med 2011;365:1663-72.

14 Bessonova L, Volkova N, Higgins M, et al. Data from the US and UK cystic fibrosis registries support disease modification by CFTR modulation with ivacaftor. Thorax 2018;73:731-40.

15 Dilokthornsakul P, Hansen RN, Campbell JD. Forecasting us ivacaftor outcomes and cost in cystic fibrosis patients with the G551D mutation. Eur Respir J 2016;47:1697-705.

16 Kirwan L, Fletcher G, Harrington M, et al. Longitudinal trends in real-world outcomes after initiation of ivacaftor. A cohort study from the cystic fibrosis registry of Ireland. Ann Am Thorac Soc 2019;16:209-16.

17 Volkova N, Moy K, Evans J, et al. Disease progression in patients with cystic fibrosis treated with ivacaftor: data from national US and UK registries. J Cyst Fibros 2020;19:68-79.

18 PCRS - Reporting Menu. Available: https://www.sspcrs.ie/portal/ annual-reporting/report/annual [Accessed 6 Aug 2020].

19 Wainwright CE, Elborn JS, Ramsey BW, et al. Lumacaftor-Ivacaftor in Patients with Cystic Fibrosis Homozygous for Phe508del CFTR. N Engl J Med Overseas Ed 2015;373:220-31.

20 Davis PB. Another beginning for cystic fibrosis therapy. $N$ Engl J Med 2015;373:274-6.

21 Taylor-Cousar JL, Munck A, McKone EF, et al. Tezacaftor-Ivacaftor in patients with cystic fibrosis homozygous for Phe508del. N Engl J Med 2017;377:2013-23.

22 Rowe SM, Daines C, Ringshausen FC, et al. Tezacaftor-lvacaftor in Residual-Function heterozygotes with cystic fibrosis. N Engl J Med 2017;377:2024-35.

23 Grasemann $\mathrm{H}$. Cftr modulator therapy for cystic fibrosis. N Engl J Med 2017;377:2085-8.

24 Heijerman HGM, McKone EF, Downey DG, et al. Efficacy and safety of the elexacaftor plus tezacaftor plus ivacaftor combination regimen in people with cystic fibrosis homozygous for the F508del mutation: a double-blind, randomised, phase 3 trial. Lancet 2019;394:1940-8.

25 Middleton PG, Mall MA, Dřevínek P, et al. Elexacaftor-TezacaftorIvacaftor for cystic fibrosis with a single Phe508del allele. N Engl J Med 2019;381:1809-19 